Submitted to the IEEE Journal of Selected Topics in Applied Earth Observations and Remote Sensing (JSTARS) Special issue on the Earth Observing One Mission - Ten Years in Space

\title{
Use of the Earth Observing One (EO-1) Satellite for the Namibia SensorWeb Flood Early Warning Pilot
}

Daniel Mandl ${ }^{1}$, Stuart Frye ${ }^{2}$, Pat Cappelaere ${ }^{3}$, Matthew Handy ${ }^{4}$, Fritz Policelli ${ }^{5}$, McCloud Katjizeu, Guido Van Langenhove ${ }^{6}$, Guy Aube ${ }^{7}$, Jean-Francois Saulnier ${ }^{7}$, Rob Sohlberg ${ }^{8}$, Julie Silva ${ }^{8}$, Nataliia Kussul $^{9}$, Sergii Skakun ${ }^{9}$, Stephen Ungar ${ }^{10}$, Robert Grossman ${ }^{11}$

${ }^{1}$ Software Systems Engineering Branch (Code 581), NASA/Goddard Space Flight Center, Greenbelt, MD 20771, USA

${ }^{2}$ SGT, Greenbelt, MD 20770, USA

${ }^{3}$ Vightel Inc., Ellicott City, MD, USA

${ }^{4}$ Ground Software Systems Branch (Code 583), NASA/Goddard Space Flight Center, Greenbelt, MD 20771, USA

${ }^{5}$ Earth Science Division (Code 610), NASA/Goddard Space Flight Center, Greenbelt, MD 20771, USA

${ }^{6}$ Namibia Hydrological Services, Windhoek, Namibia

${ }^{7}$ Canadian Space Agency, Montreal, Canada

${ }^{8}$ Univ. of Maryland, Department of Geography, College Park, MD, USA

${ }^{9}$ Space Research Institute NASU-NSAU, Kyiv, Ukraine

${ }^{10}$ Morgan State University, Baltimore, MD, USA

${ }^{11}$ University of Chicago, Chicago, Il, USA

\section{ABSTRACT}

The Earth Observing One (EO-1) satellite was launched in November 2000 as a one year technology demonstration mission for a variety of space technologies. After the first year, it was used as a pathfinder for the creation of SensorWebs. A SensorWeb is the integration of variety of space, airborne and ground sensors into a loosely coupled collaborative sensor system that automatically provides useful data products. Typically, a SensorWeb is comprised of heterogeneous sensors tied together with a messaging architecture and web services. Disasters are the perfect arena to use SensorWebs. One SensorWeb pilot project that has been active since 2009 is the Namibia Early Flood Warning SensorWeb pilot project. The Pilot Project was established under the auspices of the Namibian Ministry of Agriculture Water and Forestry (MAWF)/Department of Water Affairs, the Committee on Earth Observing Satellites (CEOS) 
/Working Group on Information Systems and Services (WGISS) and moderated by the United Nations Platform for Space-based Information for Disaster Management and Emergency Response (UN-SPIDER). The effort began by identifying and prototyping technologies which enabled the rapid gathering and dissemination of both space-based and ground sensor data and data products for the purpose of flood disaster management and water-borne disease management. This was followed by an international collaboration to build small portions of the identified system which was prototyped during that past few years during the flood seasons which occurred in the February through May timeframe of 2010 and 2011 with further prototyping to occur in 2012. The SensorWeb system features EO-1 data along with other data sets from such satellites as Radarsat, Terra and Aqua. Finally, the SensorWeb team also began to examine the socioeconomic component to determine the impact of the SensorWeb technology and how best to assist in the infusion of this technology in lesser affluent areas with low levels of basic infrastructure. This paper provides an overview of these efforts, highlighting the EO-1 usage in this SensorWeb.

\section{INTRODUCTION}

Since 2009, Namibia has experienced a surge of flooding in the Northern portion of the country. It was estimated that during 2009, 700,000 of the approximately 2 million people in Namibia were impacted by the floods of 2009, furthermore around 50,000 people were displaced and 102 people lost their lives. Estimated damage and losses during that year in Namibia were at about \$210 million U.S., which represented things such as damages to infrastructure and lost capacity in agriculture, industry, commerce and tourism [1]. Similar impacts occurred in 2010 and 2011 from additional large floods during the flood season that had begun occurring during 
the months of February through May annually. As a follow-up to the assistance provided by the United Nations Platform for Space-based Information for Disaster Management and Emergency Response (UN-SPIDER), National Aeronautics and Space Agency (NASA), Dienstleistungszentrum Ländlicher Raum (DLR), Ukraine Space Research Institute (USRI), and other space agencies in 2009, the "Namibia Flood SensorWeb” project was set up with the purpose of integrating remote sensing into a flood monitoring, and early warning decision support system. The main in country partner was the Namibian Hydrological Services. The vision was to create a decision support system oriented to local user needs, but based on a regional, trans-boundary approach, with Namibia serving as a node country. The decision support system would ingest key satellite and ground sensor data and integrate the information to provide flood situational awareness and early warning. The intent was to facilitate capacity development and institutional strengthening in Namibia to ensure the sustainable use of such a system. A collaborative proposal was developed that outlined the major components needed to build a Flood SensorWeb and a cost proposal was developed. Due to the large cost, the entire effort has thus far not been funded; however, various portions of the team have been working with the Namibian Hydrological Services to deploy portions by leveraging existing funded research along with existing resources at the Namibian Hydrological Services. This paper outlines the efforts deployed from early 2010 to the present to prototype portions of the Flood SensorWeb.

\section{BASIC FLOOD SENSORWEB ARCHICTECTURE}

[Fig 1] shows the basic SensorWeb architecture in which sensors and basic data processing services are wrapped in standardized web services interfaces and made available over the Internet. The sensor data and the higher level data products are distributed as feeds that can 
be received and discovered by users. The user can elect to provide their own portal and follow the interface standards to display or ingest the available data products. For our group, we produced the Flood Dashboard as our portal, which is hosted in the Matsu cloud. [Fig 2] shows the basic configuration of the cloud and the functional components in the cloud. The key components are the Web Coverage Processing Service (WCPS), the Flood Dashboard and recent Earth Observing 1 data. Presently, work is ongoing to also host recent Radarsat data for selected areas that are having floods such as Namibia and the Caribbean. [Fig 3] shows the basic process for using the WCPS to create customized higher level products. [Fig 4] shows the Flood Dashboard which acts as the portal to integrate the various data feeds and provides storage and display capability for key flood data. The key components that are integrated during the early 2012 time period are as follows:

(1) River gauges in the various river catchments with telemetered data

(2) TRMM rainfall and global flood estimate maps

(3) Daily MODIS flood extent maps

(4) Earth Observing 1 Advanced Land Imager (ALI) and Hyperion data and its derived flood extent maps

(5) Radarsat Synthetic Aperture Radar (SAR) imagery with derived flood extent maps

(6) Global Disaster and Coordination System(GDACS) based on the AMSR-E microwave instrument data originating from the Terra satellite, to estimate river flows

(7) An experimental Coupled Routing and Excess STorage (CREST) model developed by the University of Oklahoma for flood modeling

Many of the components are in experimental stages. For example, the CREST model still needs to be further calibrated to be useful in Namibia. The team plans to do this extra calibration if 
funding becomes available. The Radarsat data has been placed in the Matsu Cloud and can be displayed as a layer. Workflow scripts enable users to orchestrate the various scenarios to task assets such as EO-1 and then to deliver the products to the Flood Dashboard. EO-1 tasking and data products delivery is mostly automated and the complete data processing chain is planned to be complete in 2012. The team is beginning to work on a similar tasking and data delivery process for Radarsat. Work has begun to define this process within constraints of budget and issues for licensing of the data.

\section{KEY ACTIVITIES TO INEGRATE THE FLOOD SENSORWEB}

[Fig 5] shows one of the key flood prone areas in Northern Namibia along with two of the larger towns. Inset pictures in the map provide a visual depiction of the towns from the air. Heavy rains to the north in Angola, eventually make their way downstream and flood the mostly flat areas which are heavily populated. [Fig 6] shows the Flood SensorWeb components applied to gather key data. Note that the Flood Dashboard, in essence subscribes to the key data. Users can access the data with their own portal if they understand the interface standards. The intent is to make the interface open and accessible as possible. The data access interface for EO-1 is open to anyone, while the tasking interface for EO- 1 is limited to people who have been authenticated as valid users. However, those validated users access tasking functionality via the open Internet.

Key to making the Flood SensorWeb useful is the timeliness and accuracy of the data. The Flood SensorWeb team has worked closely with the Namibian Hydrological Services (NHS) to refine the SensorWeb to make it useful. This means that just integrating the sensor data feeds 
and displaying maps is not sufficient. The team has visited Namibia three times, January 2010, 2011 and January 2012. Key issues addressed in the list of priorities were:

(1) Timely access and data product creation of satellite data

(2) Satellite data processing techniques and training for in country hydrologists

(3) Validation of satellite data in terms of accurately identifying water locations

(4) Understanding key social issues that are obstacles to technology infusion

The issue of timeliness was one of key issues articulated by the Namibia Hydrological Services. To that end, the majority of the work conducted is to provide faster and easier access. The team provided tasking capability of Earth Observing 1 to NHS via the GeoBPMS, also known as the Campaign Manager as shown in Figure 1. Training was provided to NHS to process the EO-1 images into water classification maps [Fig 7]. Furthermore Rob Sohlberg, at Univ. of Maryland, is experimenting with the Weka data mining tool and the Web Coverage Processing Service (WCPS), to create a muddy/clear water classifier algorithm that will be installed in the cloud to automatically generate EO-1 Advanced Land Imager based water extent maps. Once initial training is complete for NHS and the algorithm generated by Rob Sohlberg is complete and operational within the Matsu cloud environment, the desire is to have NHS along with students from University of Namibia use the Web Coverage Processing Service (WCPS) to augment and create new algorithms to apply to EO-1 data and to create new data processing pipelines. Thus the students and hydrologists will be customizing their own experience to add new data layers for EO-1 data.

Recent exercises already point to some of the derived products created by NHS. For example, [Fig 8] shows a map created by McCloud Katjizeu in collaboration with the Ministry of 
Health and the SensorWeb team to experiment with the use of the water extent maps to aid in the application of larvacide for mosquito and thus Malaria control. This effort was initiated to begin to showcase the decision support capability of the system and to make a compelling case for its use. The team continues to examine issues related to water and health to infuse SensorWeb data to aid in additional decisions. In another example, NHS used EO-1 imagery to assist Walvis Saltworks in Swakopmund in monitoring the influx of ocean water into their salt fields. These fields must be carefully monitored for breeches in the barrier between the fields in which salt is produced by drying out ocean water and the actual ocean.

The more challenging task is the streamlining of Radarsat sensor data. The vision is to use the GeoBPMS to identify an area of interest which would subsequently task EO-1 and Radarsat. Furthermore, the team will attempt to automate the data production pipeline for Radarsat to the degree possible to create map layers in a rapid manner. Work to make this happen is ongoing at the writing of this paper.

Initial experiments were conducts in 2011 and early 2012 coincident with visits to the Divindu area of the Kavango in Northern Namibia. [Fig 9] \& [Fig 10] show some initial images processed by Canadian Space Agency (CSA). [Fig 9] is an image of the Kavango in March 26, 2011 and shows more black (open water) than the [Fig 10] which was imaged of the same area in January 24, 2012. One of the key issues is the calibration of Radarsat data and it was noted by CSA that it may be incorrect that the water extent is higher in 2012 because Radarsat may be reflecting different vegetation conditions at the time of the imagery due to seasonal vegetation variations. This exemplified one of the key challenges to the team for calibrating of the radar data to create a differential map showing unusual water pattern changes indicating flooding. 
To understand some of these issues, NHS conducted some ground validation exercises using the SensorWeb team visit during the last week of January 2012. Control points were marked with GPS and inserted into Radarsat data products to conduct validation exercises. [Fig 11] shows one if the experimental Radarsat data products that was created by McCloud Katjizeu. Thus it represented both training and research for NHS as to how well Synthetic Aperture Radar (SAR) could be used to identify water locations.

One key activity is the training and development of better hydrological models to aid in predicting flood waves and thus provides early warning. At this time, Guido Van Langenhove, Head of NHS, along with his staff, monitor the various data feeds and request the needed data to formulate a model in their mind for the arrival of flood waters. There is a desire on both NHS and the SensorWeb team to capture this modeling knowledge and to automate to the degree possible for early warning alerts. Linkages in the SensorWeb have been established as experiments to automate the flow of information which is needed to populate a model of this kind. For example, the Global Disaster and Coordination System (GDACS) monitors rivers north of Namibia with both AMSR-E and TRMM. When preselected rivers north of Namibia exceed a given threshold in term of river width, automatic triggers to the GeoBPMS are provided that task EO-1 and in the future Radarsat to provide downstream high resolution images of the target towns. The Flood Dashboard provides the capability to trigger the automatic tasking of satellites via a variety of triggers, starting with GDACS and perhaps using the CREST model or upstream river gauges. As the usage of these capabilities increase, the decision assistance will become more complex and can be used to assist NHS hydrologists in many activities. The key 
challenge is capturing the complexities of the terrain and the knowledge of the hydrologists employ, to make key decisions.

Another experimental product, created by Ukraine Space Research Institute, was a flood hazard and flood risk assessment map which made use of multiple satellite data sources. The approach used was to analyze a time-series of Landsat TM/ETM+ images along with EO-1 data to estimate flood probability density. As a first step, clouds, shadows and SLC-off pixels (for the ETM+ instrument) were identified on Landsat scenes; marked as a "No Data" value, and removed from the further analysis. For the second step, water bodies are detected using a density sliding method. Therefore, each pixel was given one of the following values: 0 - "no water", 1 “water” or 2 - "no data”. Landsat scenes were co-registered and aggregated to produce an image showing the Probability of Inundation (PI) value [Fig 12].

The final activity that was initiated during the team trip in January 2012 was to examine how to measure the Sensorweb technology impact at a social or economic level [Fig 13]. Visits to villages adjacent to the Kavango River were conducted by the SensorWeb team to determine the viability of conducting socio-economic surveys in the future to determine the effectiveness of technology infusion. The government of Namibia has already created a Post Disaster Needs Assessment in 2009 and periodically updates this document and considers this document as the basis to beginning to analyze the potential impact of the Flood SensorWeb technology.

\section{CONCLUSION}


The SensorWeb activity is a continuous improvement exercise. The software architecture is designed similarly to the Internet so that as new data, data products and models emerge, they can be integrated into the architecture seamlessly and then easily accessed by users.

\section{FIGURES}

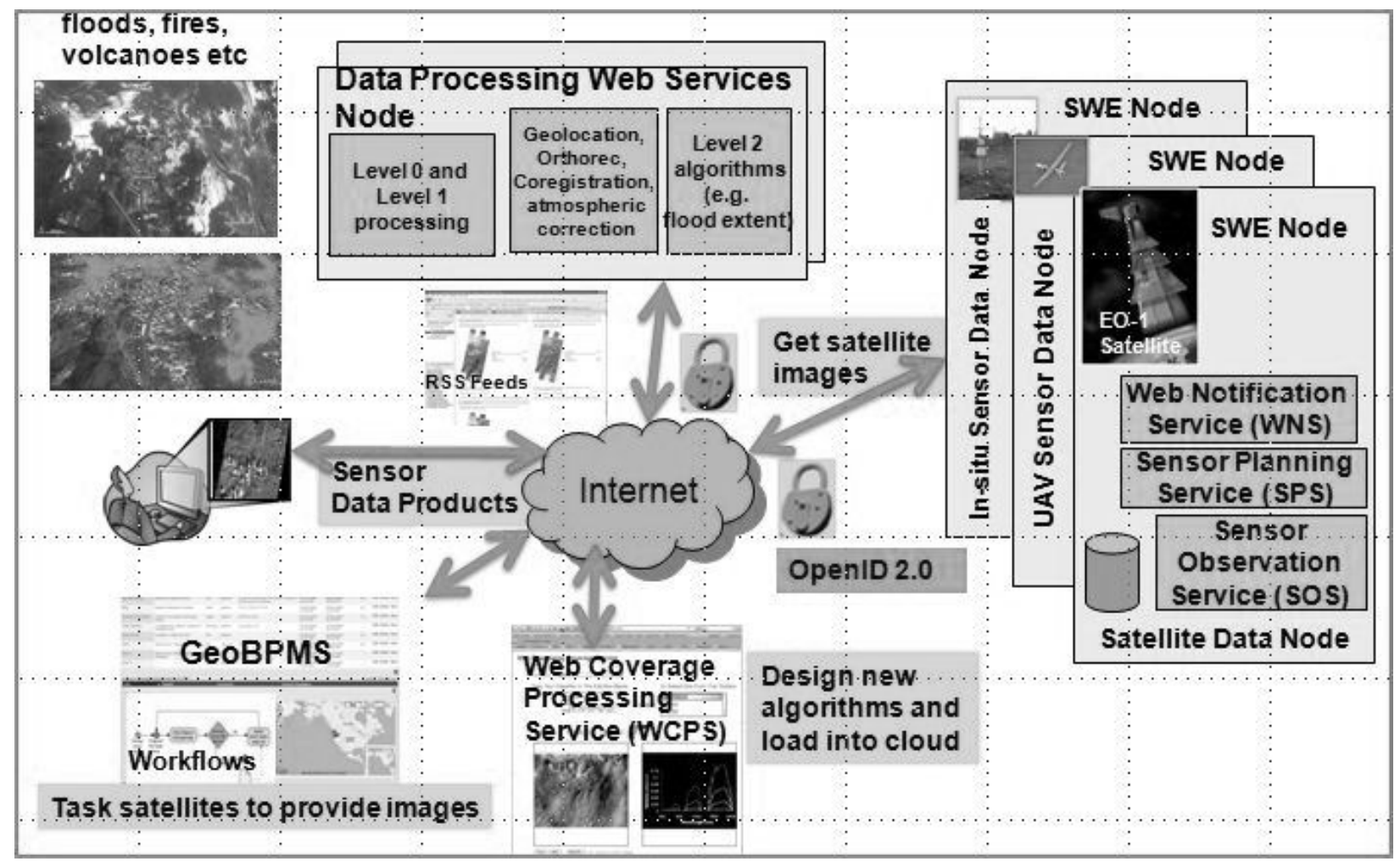

Fig. 1. High level picture of SensorWeb architecture showing the use of web services to create sensor data feeds 


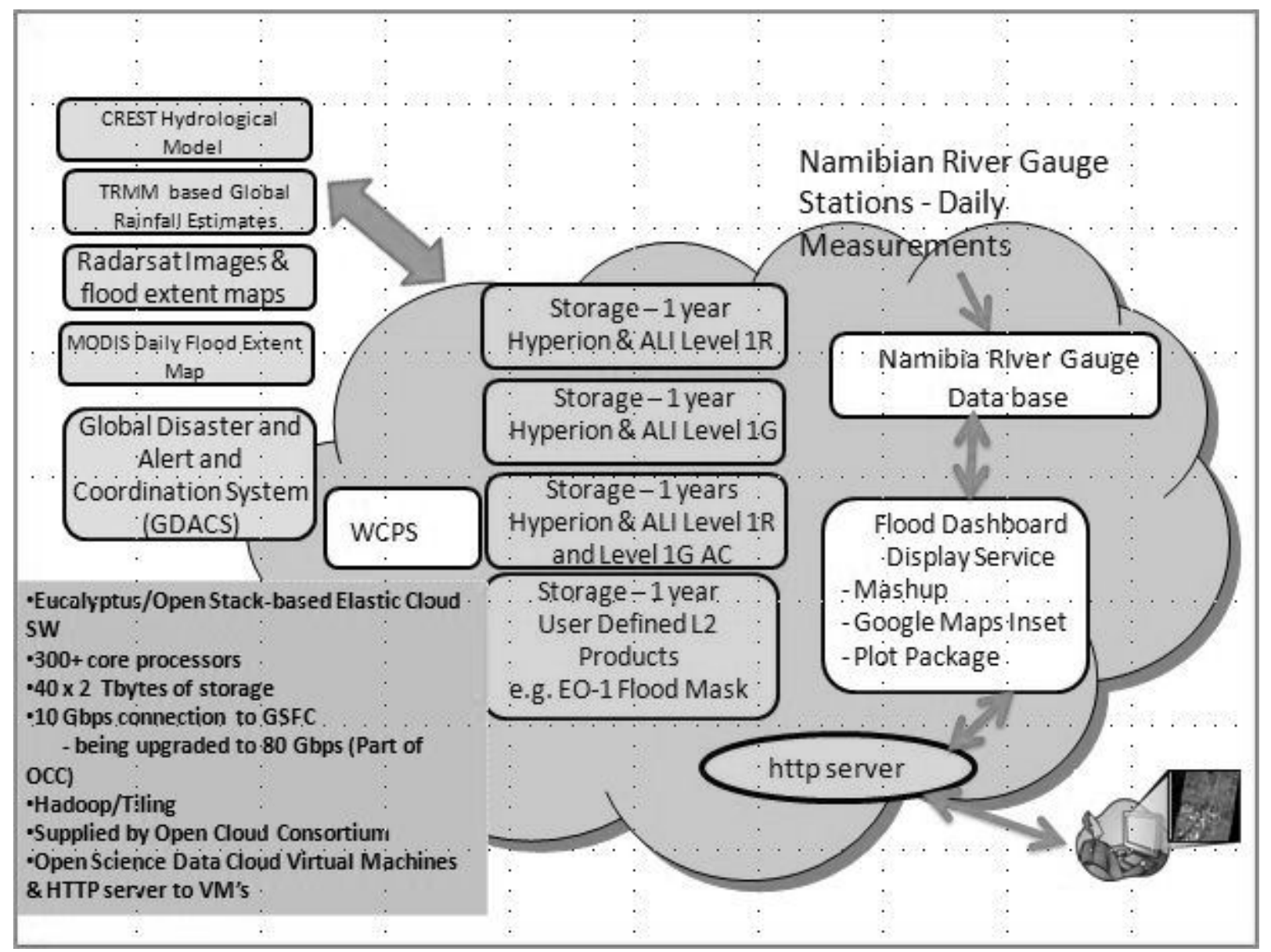

Fig. 2. Matsu cloud being used to host the Web Coverage Processing Service (WCPS), the Flood Dashboard and the latest data from Earth Observing 1 data 


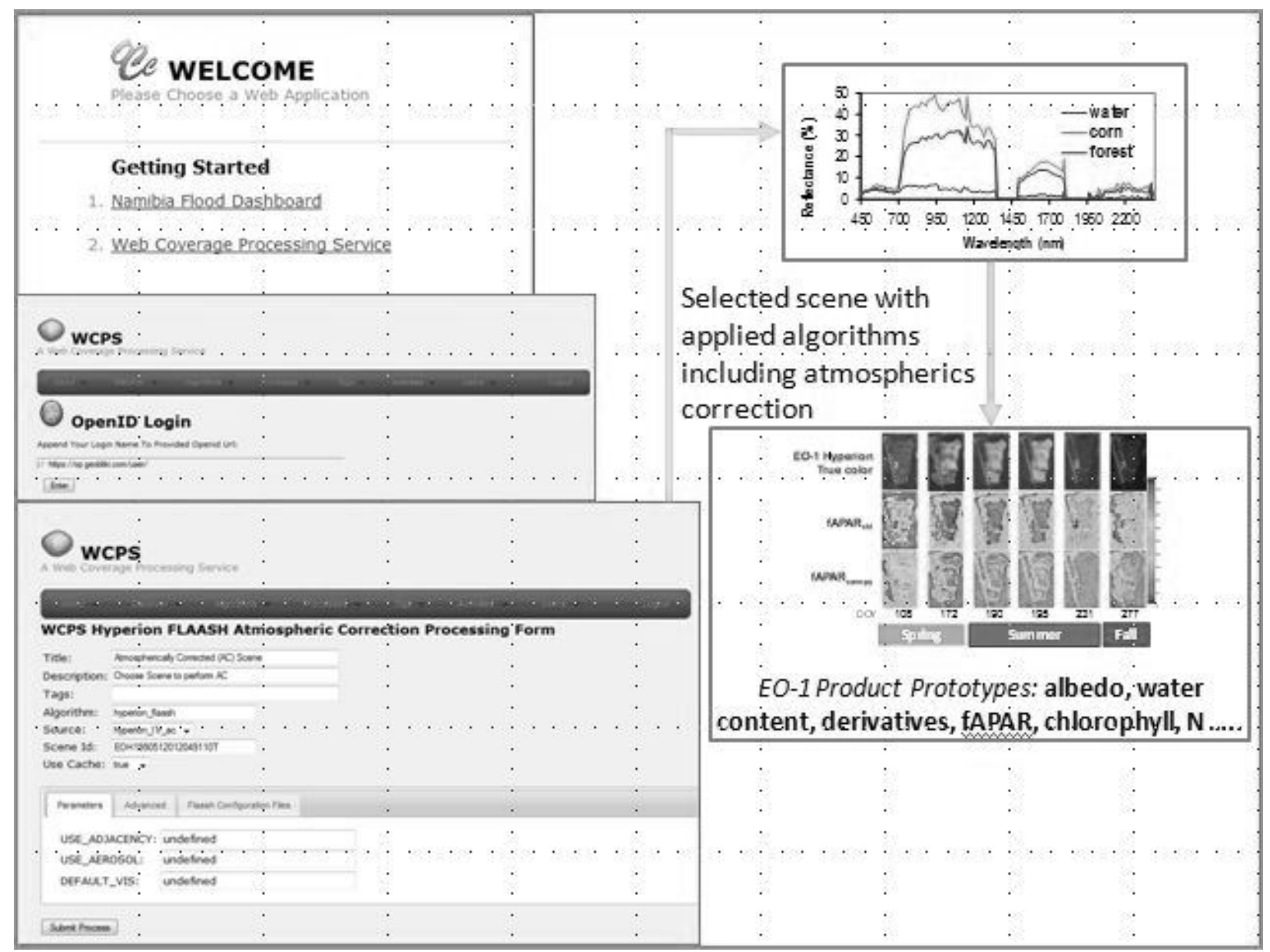

Fig. 3. WCPS process to apply atmospheric corrections and other user-defined algorithms to create customized EO-1 data products such as flood extent maps 
12 HYDROLOGICAL SERVICES NAMIBIA - DAIIY FLOOD BULLETIN 19 FEBRUARY 2012 - MORE GOOD RAINS IN CATCHMENTS OF NORTHERN RIVERS AND NEW FLOODS IN LOWER FISH RIVER

View Complete Curent Bulletin

View Bulletin Records

Search Bulletin Records

New Bulletin

\begin{tabular}{|l|}
\hline$\nabla$ River Stations \\
\hline$\nabla_{\text {SensorWeb Layers }}$ \\
\hline$\nabla_{\text {Water Lines and Areas }}$ \\
\hline$\nabla_{\text {Satelite Overlays }}$ \\
\hline$\nabla_{\text {Ground Pics }}$ \\
\hline$\nabla_{\text {Kavango Radarsat Data }}$ \\
\hline$\nabla_{\text {Cuvelai Radarsat Data }}$ \\
\hline$\nabla_{\text {TRMM Rainfall Accunulation and Flood }}$ \\
Forecast \\
\hline$\nabla_{\text {Global Scene Counts }}$ \\
\hline$\nabla_{\text {MODIS Floodmaps }}$ \\
\hline$\nabla_{\text {Infrastructure }}$ \\
\hline$\nabla_{\text {ALI Flood Classification }}$ \\
\hline
\end{tabular}

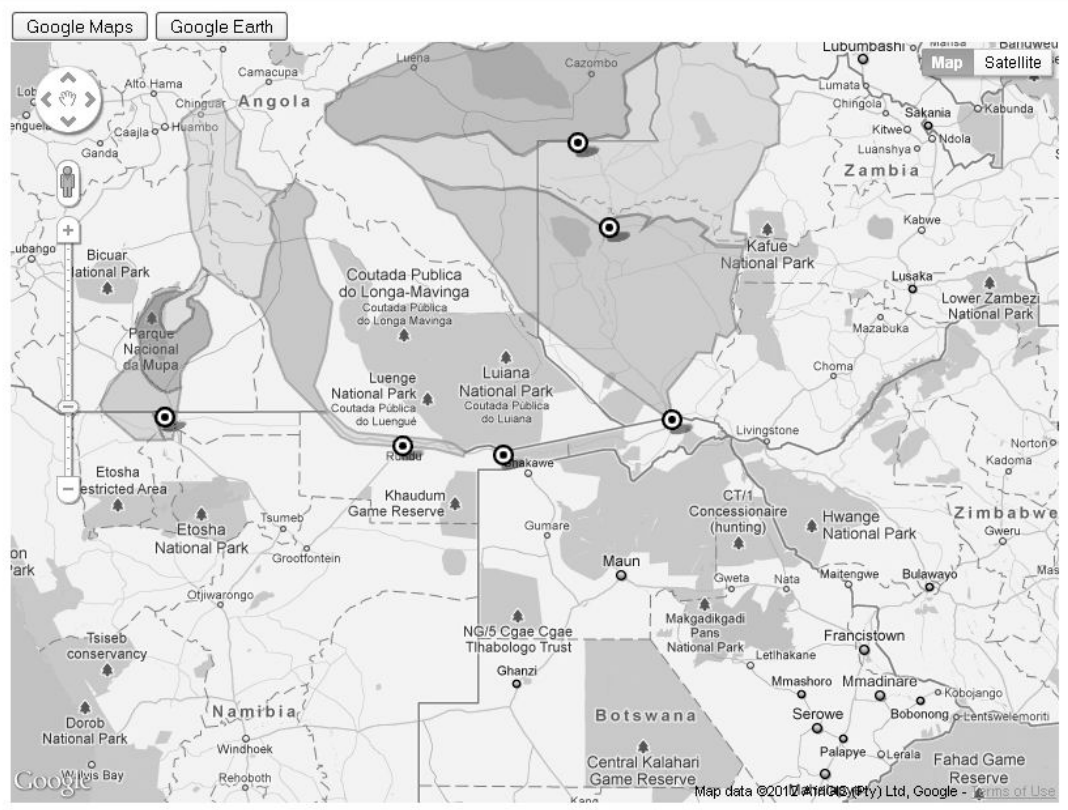

Fig 4. Flood Dashboard which acts as portal for key flood data and provides display and mapping capability 


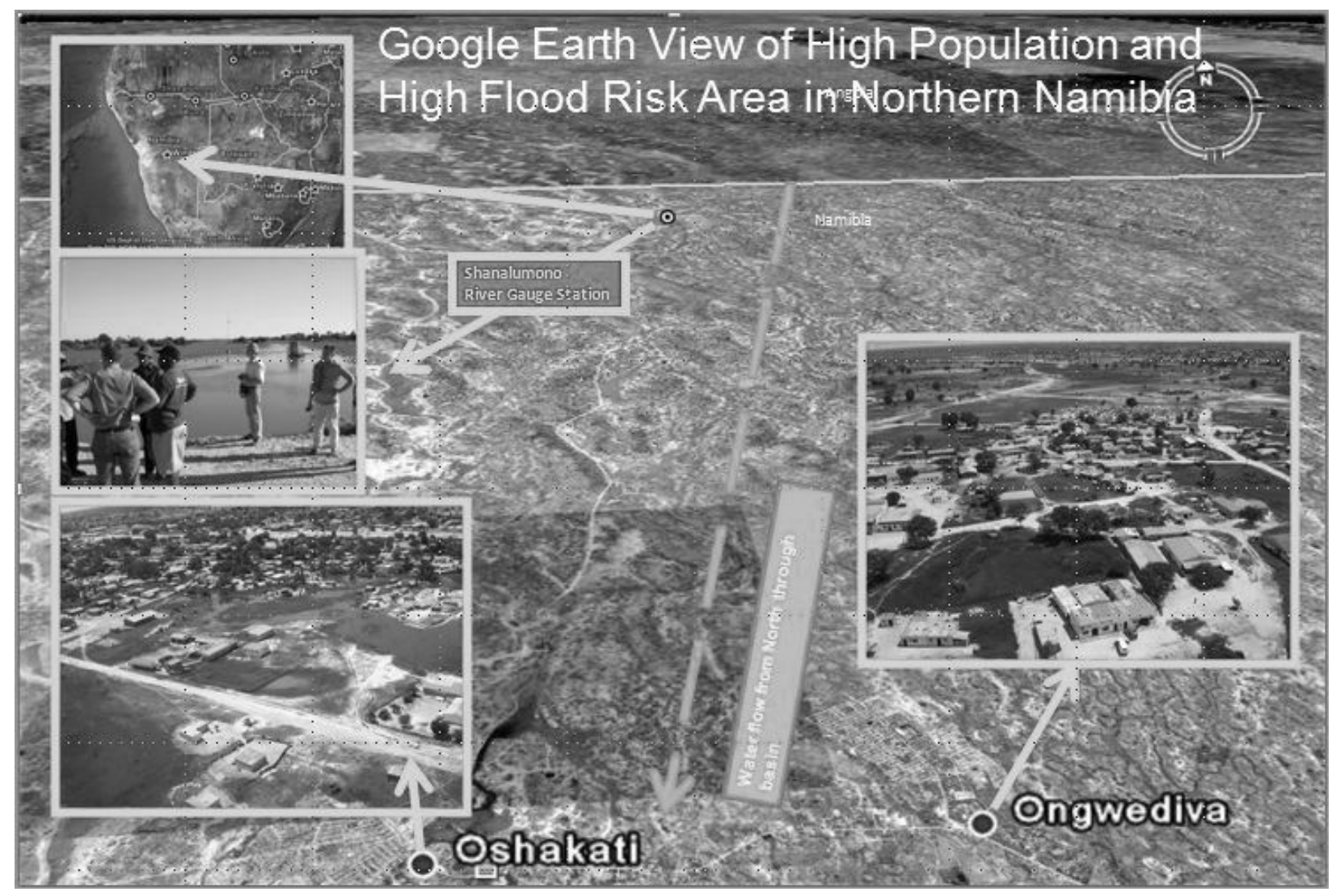

Fig. 5. One of the high flood risk areas in Northern Namibia 


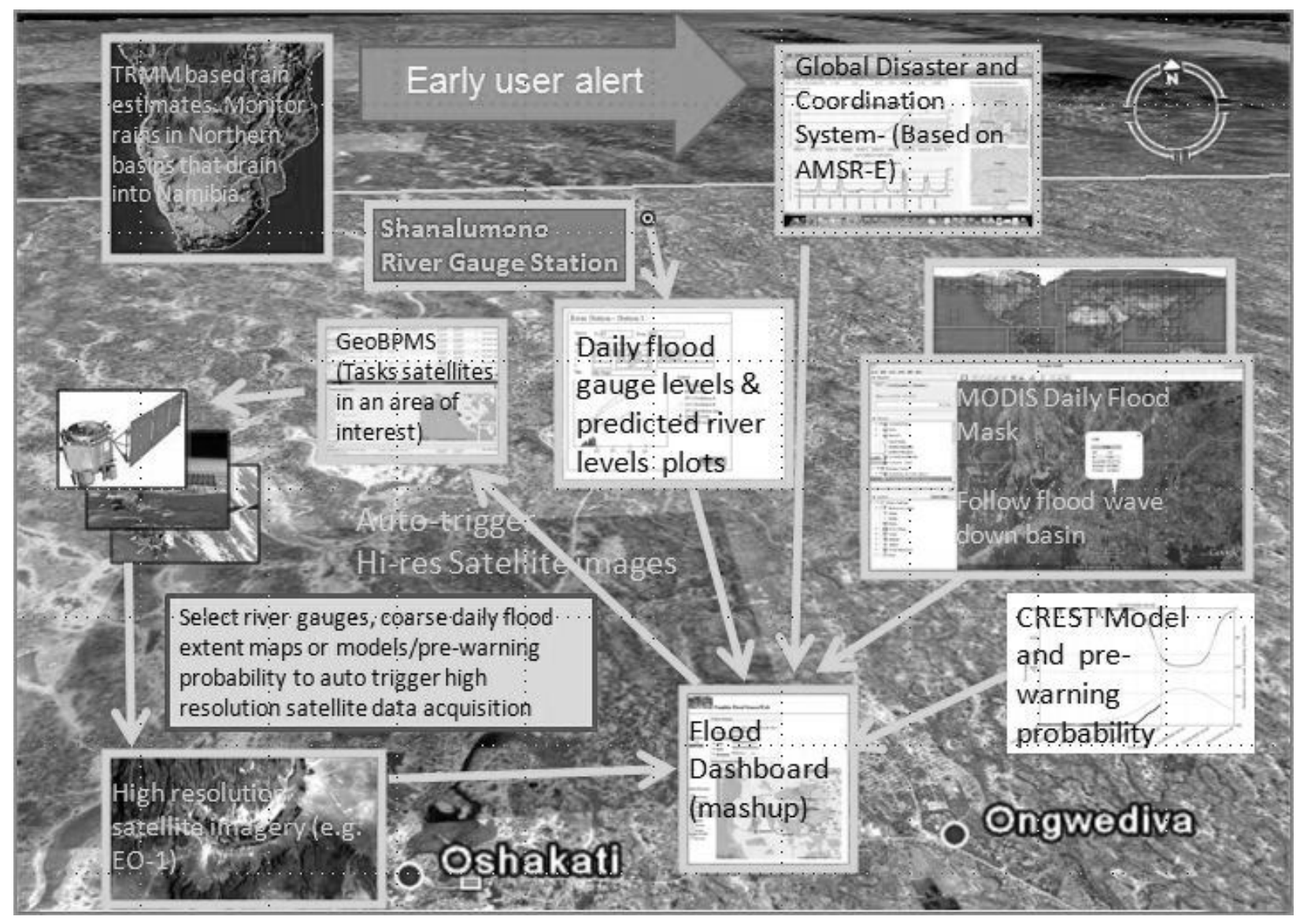

Fig. 6. Components linked to Flood Dashboard for Flood SensorWeb for Northern Namibia

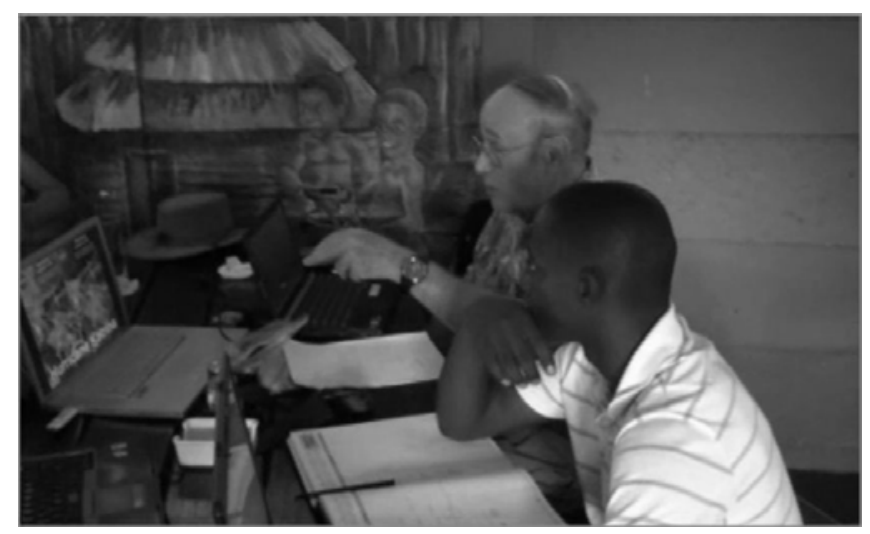

Fig. 7. Dr. Stephen Ungar/NASA training McCloud Katjizeu/NHS hydrologist on techniques to process EO-1 data during the team's visit in January 2011 


\section{Flood Map}

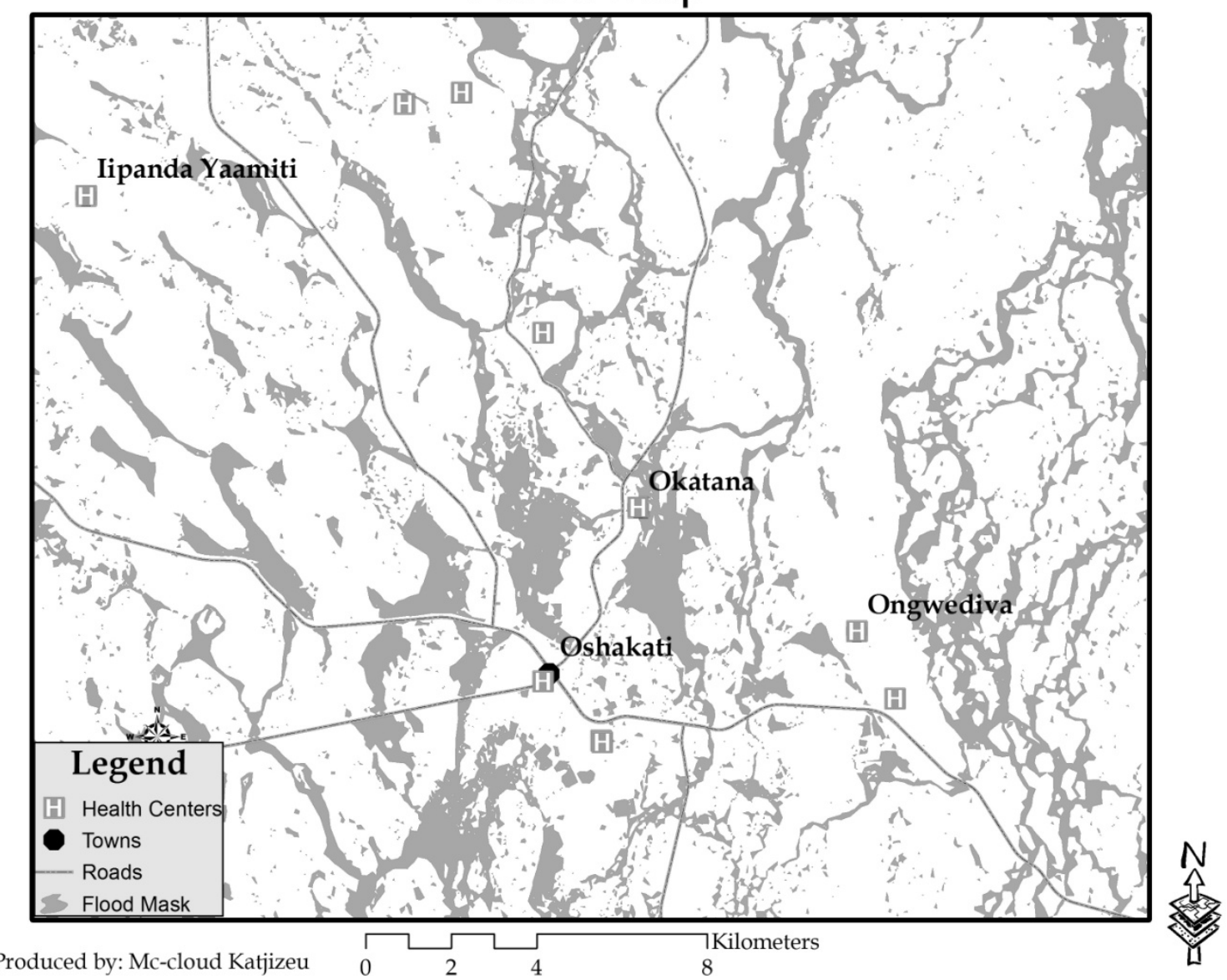

Fig. 8. Map created by McCloud Katjizeu/NHS using EO-1 data and Geographical Information System (GIS) data for locations of roads, towns and health centers. Exercise conducted in collaboration with Ministry of Health, Vector Control Program to experiment with maps to aid in mosquito/Malaria control by identifying standing water to apply larvacide at key times during the year. 


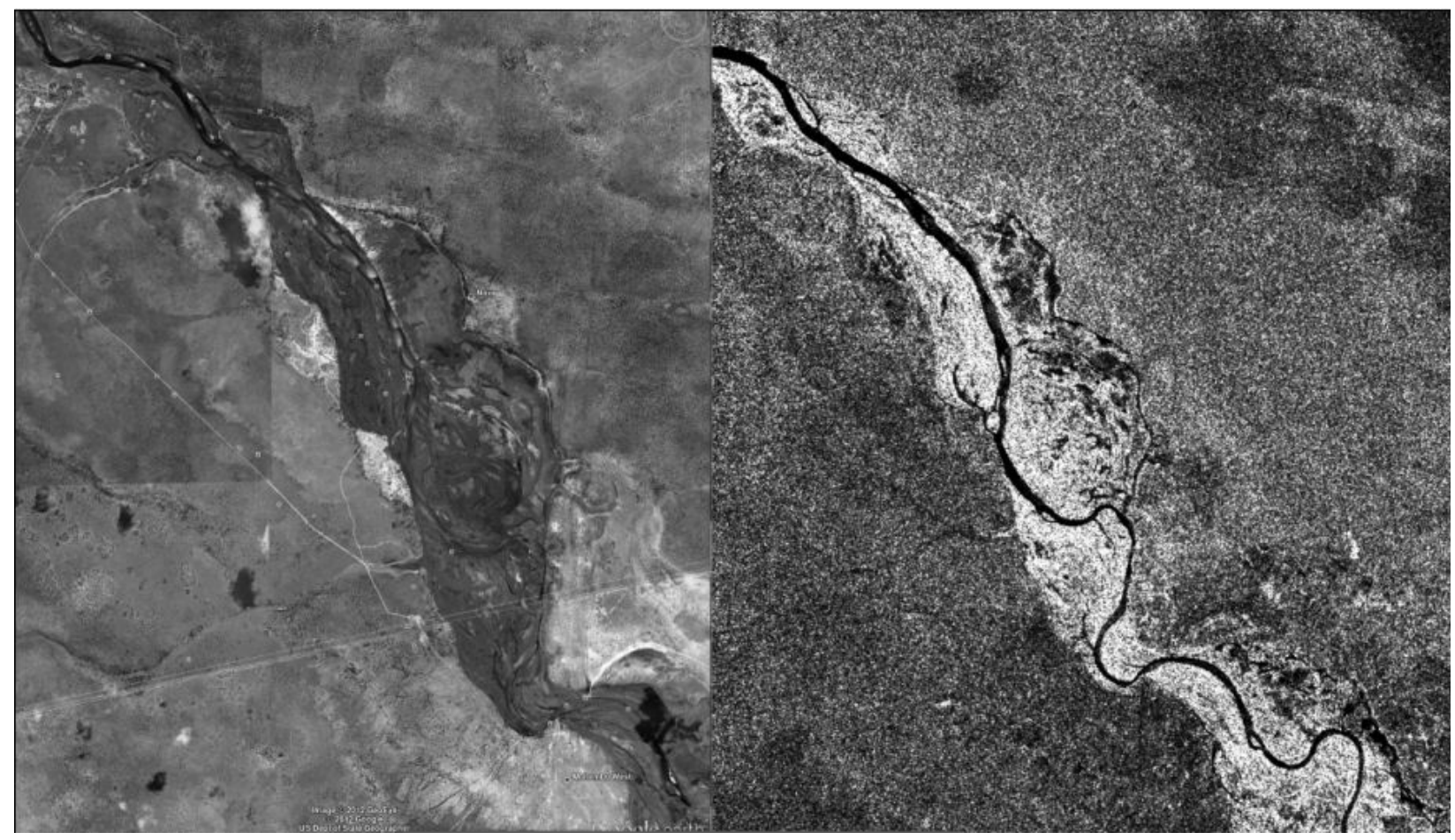

Fig. 9. Radarsat processed image from March 26, 2011 in right panel compared to Google Earth image in left panel.

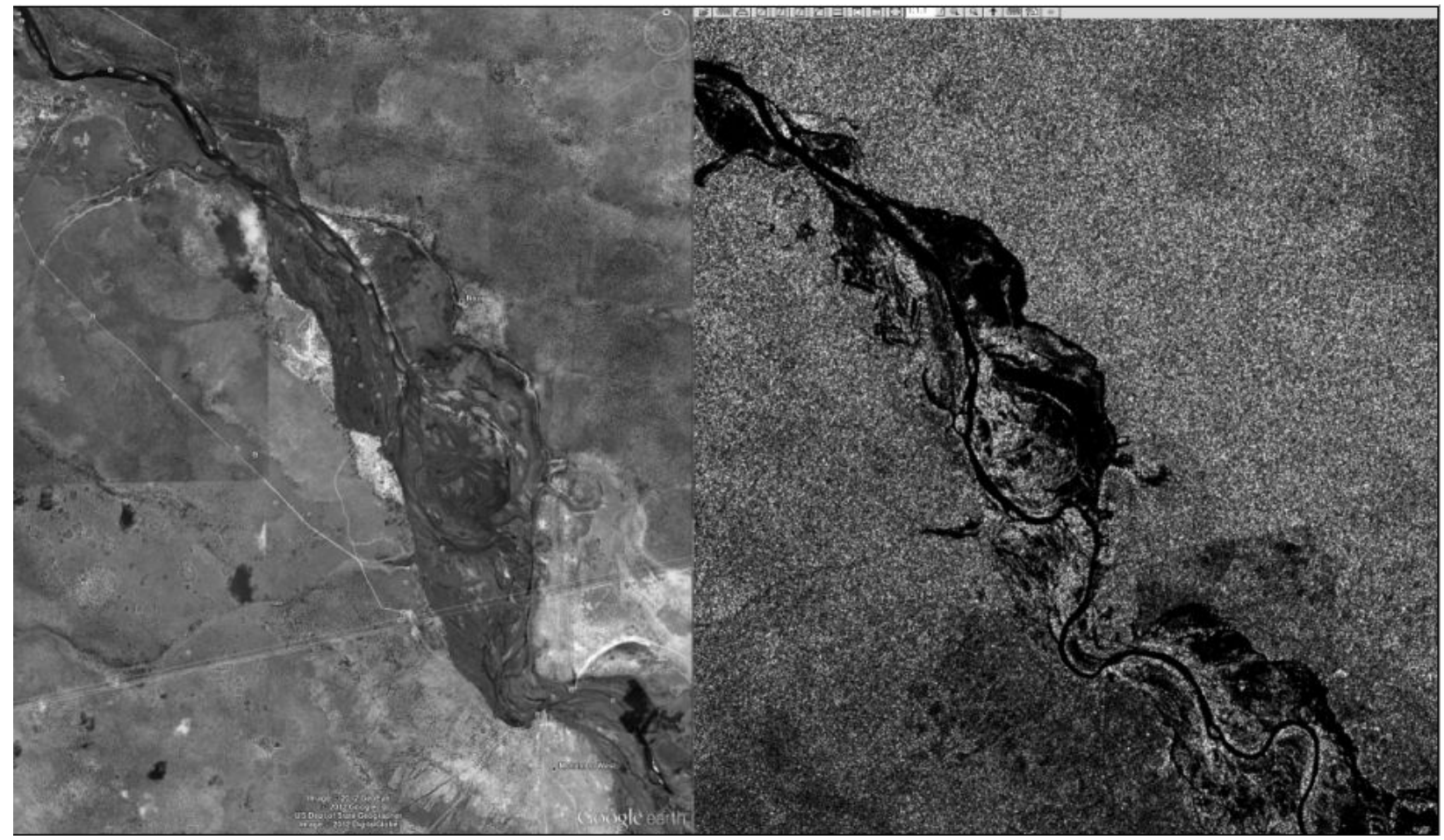

Fig. 10. Radarsat processed image from January 24, 2012 in right panel compared to Google Earth image in left panel. 


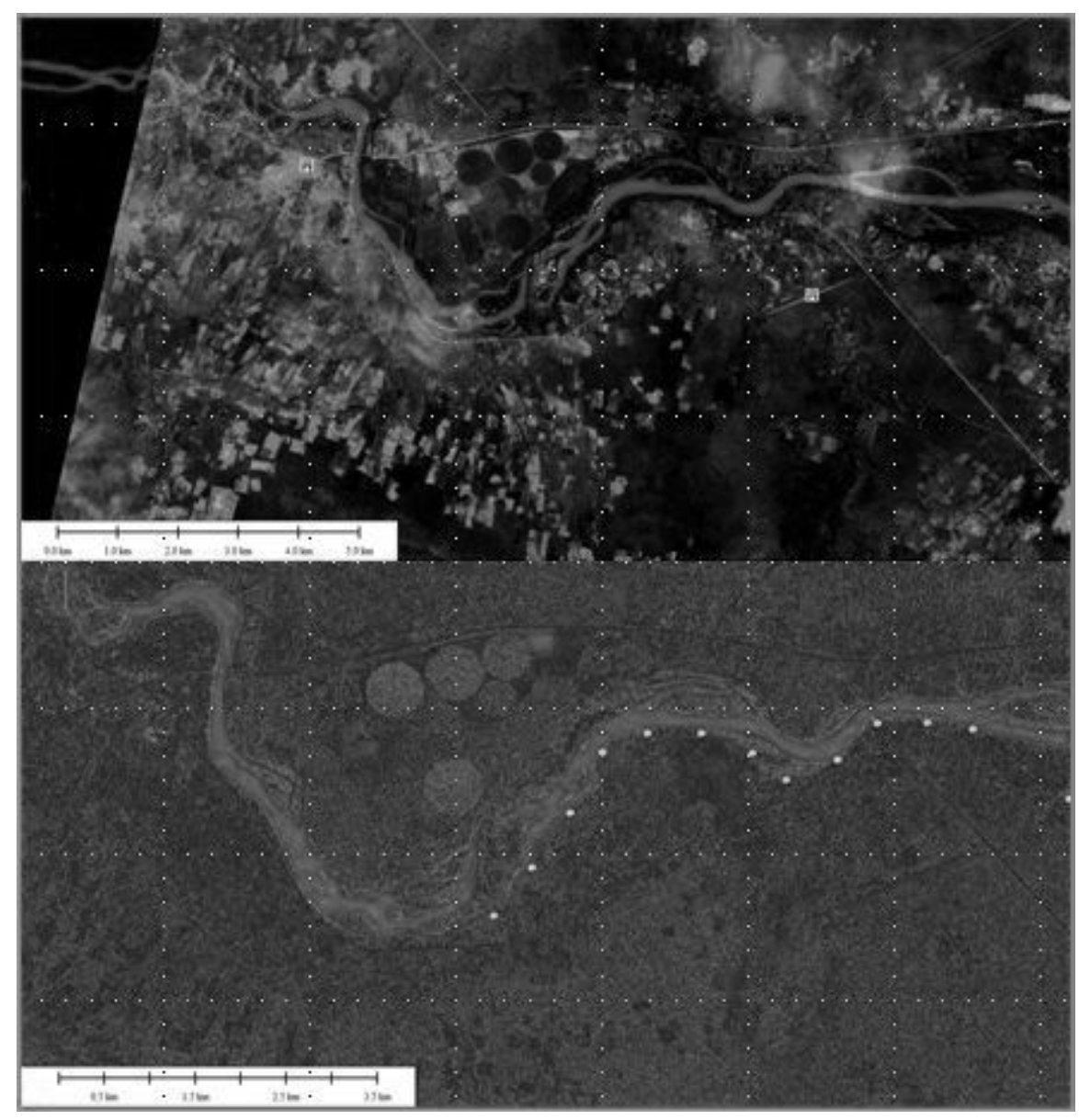

Fig. 11. Experimental image using Radarsat image taken approximately January 23, 2012 with control points displayed used to validate water location. Top panel is a rectified overlay of Radarsat located water on top of EO-1 image taken on January 24, 2012. Created by McCloud Katjizeu/NHS 


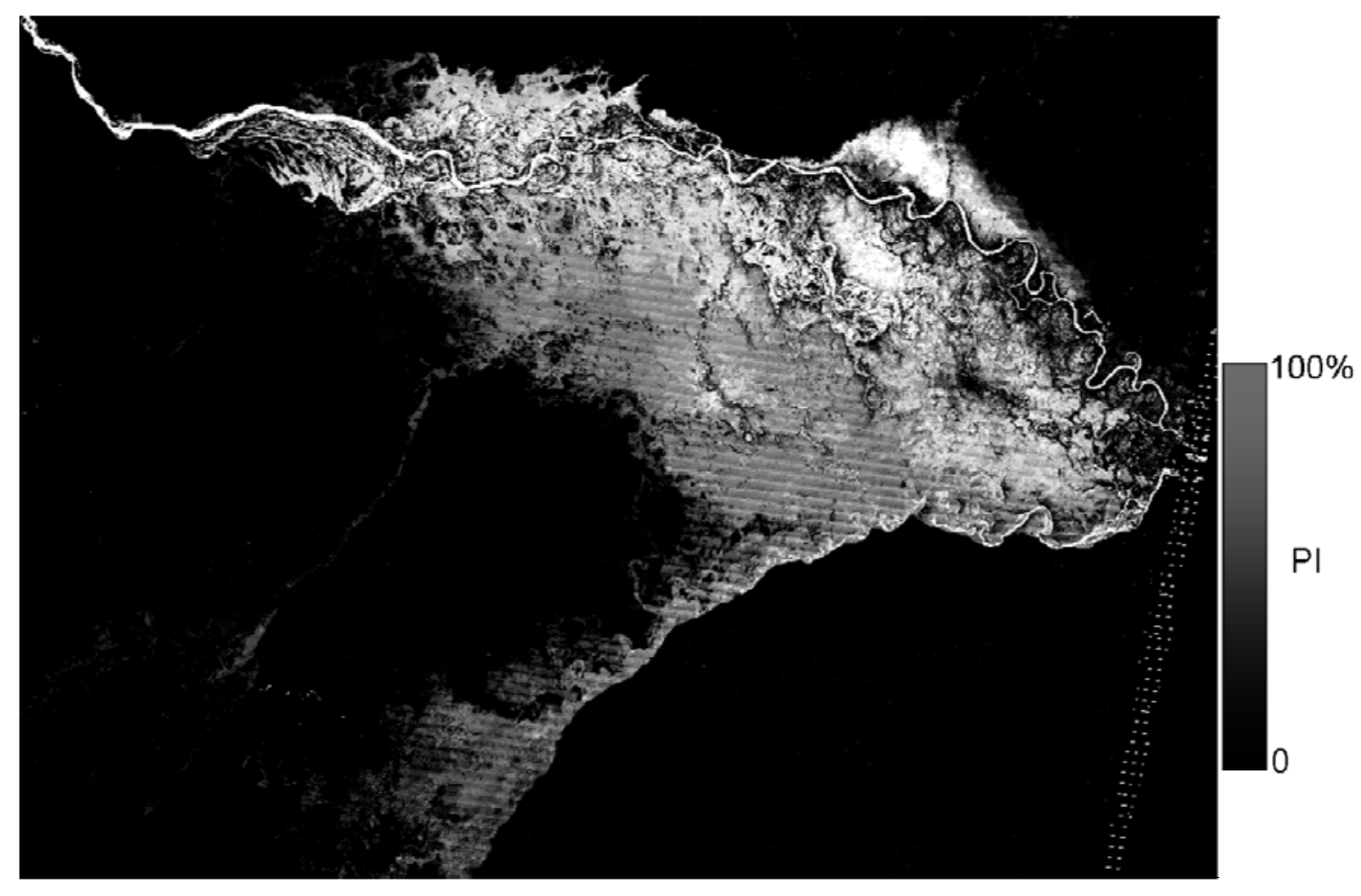

Fig. 12. Probability of inundation (PI) for the Katima Mulilo region, Namibia created by Ukraine Space Research Institute

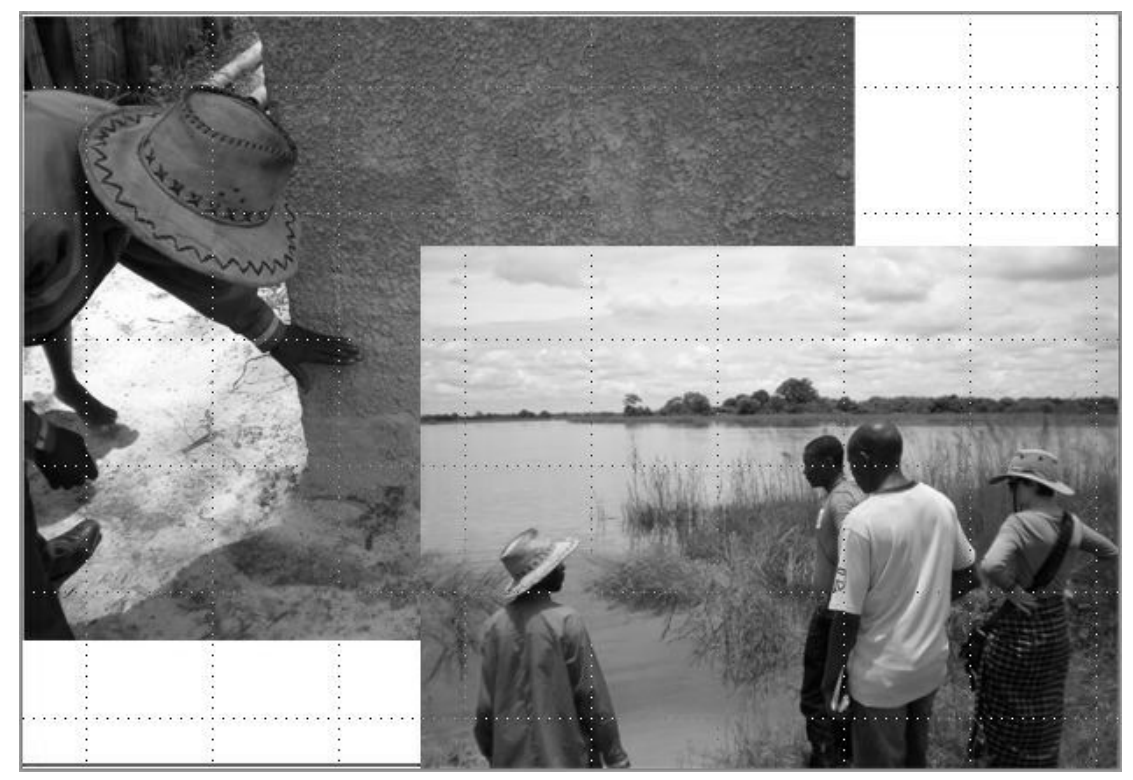

Fig. 13. Preliminary visits conducted to villages adjacent to Kavango to investigate potential Flood SensorWeb technology impact 


\section{ACKNOWEDGEMENTS:}

The authors would like to acknowledge the effort and accommodations provided to the SensorWeb team by the Namibia Hydrological Services in this collaborative effort.

\section{REFERENCES:}

[1] “Post Disaster Needs Assessment 2009”, A Report Prepared by the Government of the Republic of Namibia, with support from the International Community

[2] [4] Mandl, D., Frye, Policelli, Cappelaere, “ An International Disaster Management SensorWeb Consisting of Space-based and In-situ Sensors”, AGU, December 2009, San Francisco, CA

[3] Mandl, D., “Namibian Flood Early Warning SensorWeb Pilot”, AGU, December 15, 2010 San Francisco, CA

[4] M. Handy, "Matsu: An Elastic Cloud Connected to a SensorWeb for Disaster Response”, Cloud Computing and its Applications (CCA), Argonne National Lab, Argonne, IL, April 2011 